\title{
Choroidal haemodynamics in glaucoma
}

Glaucoma Center, Department of Ophthalmology, Academic Medical Center, University of Amsterdam, Netherlands H F A Duijm

E L Greve

Department of Medical Physics, University of Amsterdam, Netherlands H F A Duijm T J T P van den Berg

The Netherlands Ophthalmic Research Institute, Amsterdam, Netherlands

H F A Duijm

T J T P van den Berg

Correspondence to: H Ferdinand A Duijm, Glaucoma Center, Department of Ophthalmology, AMC, University of Amsterdam, PO Box 22700, 1100 DD Amsterdam, Netherlands.

Accepted for publication 14 May 1997

\author{
H Ferdinand A Duijm, Thomas J T P van den Berg, Erik L Greve
}

\begin{abstract}
Aim-Quantification of haemodynamics of the peripapillary choroid in and the assessment of possible differences between normal subjects $(\mathrm{N})$, ocular hypertensive (OHT), primary open angle (POAG), and normal pressure glaucoma (NPG) patients.

Methods-Video fluorescein angiograms (Rodenstock SLO 101) were made in $22 \mathrm{~N}$ subjects, 12 OHT, 48 POAG, and 46 NPG patients. The angiographically derived dye build up curves were described by means of an exponential model. One of the model parameters is the time constant $\tau$ theoretically reflecting local blood refreshment time; the blood refreshment time $\tau$ is the time needed to replace the blood volume in the choriocapillaris, inversely proportional to the local choroidal blood flow. Other variables are maximal fluorescence $\left(F_{\mathrm{dt}}\right)$ and time of first fluorescence $\left(t_{0}\right)$. Mean variable values were calculated for disc area and circular areas around the disc.
\end{abstract}

Results- $F_{\mathrm{dt}}$ of the disc was significantly lower in the POAG and NPG patients. There was no statistical difference in $t_{0}$ between the study groups. The choroidal blood refreshment time was significantly longer in NPG patients and to a lesser extent in the POAG patients compared with the normal controls. The slowest choroidal blood refreshment can be found in the NPG group. The median choroidal blood refreshment times (25th-75th percentile) in the controls, OHT, POAG, and NPG patients were 4.1 (3.7-4.5), 4.4 (3.7$6.4), 5.8(4.3-6.8)$, and 7.1 (5.5-9.3) seconds respectively.

Conclusions-With the help of parametrisation of dye curves, using a one compartmental model, choroidal haemodynamics can be quantified. The blood refreshment time of the peripapillary choriocapillaris was found to be significantly prolonged especially in NPG patients; this may indicate slower choroidal haemodynamics in NPG patients.

(Br f Ophthalmol 1997;81:735-742)

A vascular role in the pathogenesis of primary open angle glaucoma (POAG) has been postulated for many years. ${ }^{1-3}$ Evidence is accumulating that a disturbance of blood flow in the posterior pole does exist, especially in patients with normal pressure glaucoma (NPG) with intraocular pressures within statistically normal limits. In some POAG patients, possibly secondary, haemodynamic changes may exist as well.

Several methods to evaluate blood flow in the posterior pole have been developed. Some are restricted, because of their destructive or invasive nature, to experimental studies in animals such as methods using radioactively labelled microspheres. ${ }^{4}$ Other methods are applicable in humans - for example, methods estimating retinal capillary flow using either the laser speckle phenomenon ${ }^{5}$ or the blue field entoptic phenomenon, ${ }^{6}$ and methods estimating the pulsatile component of the ocular blood flow. More recently, colour Doppler imaging of various larger vessels-for example, ophthalmic artery, central retinal artery, and short posterior ciliary arteries, has been introduced, showing blood flow velocities in these vessels. ${ }^{7}$ Using another technique also based on the Doppler principle, the bidirectional laser Doppler velocimetry, retinal, choroidal, and optic nerve head blood flow has been measured in humans. ${ }^{8-10}$ Combining scanning laser technique and Doppler analysis, the scanning laser Doppler flowmeter (the Heidelberg retina flowmeter) aims at measuring flow in retinal capillaries. ${ }^{11} 12$

Angiography is another important technique for the assessment of blood flow. Ever since the introduction of fluorescein angiography the retinal circulation especially has been studied extensively, mostly by qualitative means. Various methods have been developed to quantify retinal circulation by photographic means $\mathrm{s}^{13-15}$ and, in recent times, using video techniques and computerised image analysis. ${ }^{16-18}$ In general, these methods aim to quantify retinal circulation by data which are derived from fluorescence intensity curves. In other words, from the shape of the dye curves information on rheology is inferred-for example, passage or circulation times and arterial dye velocities.

Attempts have been made to quantify choroidal circulation using indocyanine green angiography and image processing. ${ }^{19-21}$ In these methods the pulsatile character of the choroidal blood flow is described. As in the retinal fluorescein angiographical methods, with the aid of indocyanine green, arterial, venous and capillary filling times in the choroid can also be estimated. $^{22}$

Fluorescein has also been used successfully to study choroidal haemodynamics. A vast amount of qualitative knowledge has been obtained on physiological, normal choroidal blood flow, ${ }^{23-27}$ as well as on pathological conditions such as glaucoma. ${ }^{1-3}$ 28-30

In an attempt to describe and analyse choroidal capillary blood flow, a rheological model, describing the filling of the choriocapil- 


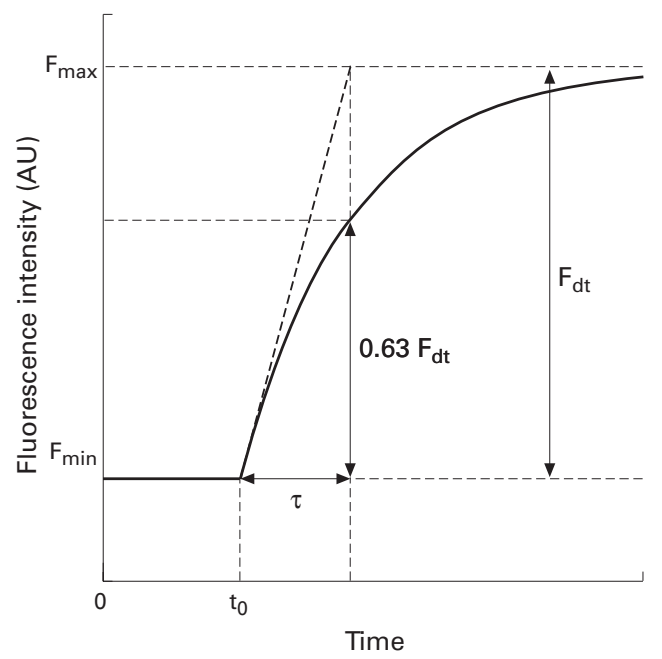

Figure 1 The graphical representation of the model curve used to analyse the individual dye build up curves. The time course is described by four variables - initial fluorescence $\left(F_{\text {min }}\right)$, maximum fluorescence $\left(F_{d}\right)$, the time of first dye appearance $\left(t_{0}\right)$, and tau $(\tau)$, the time constant of the model, representing the refreshment time

laris during fluorescein angiography, was used to analyse fluorescence intensity curves. ${ }^{31-33}$ For each location of the posterior pole the dye build up curve is analysed using the following model:

$F(t)= \begin{cases}F_{\min } & ; 0<t<t_{0} \\ F_{d t} \cdot\left(1-e^{\frac{-\left(t-t_{0}\right)}{\tau}}\right)+F_{\min } & ; t \geqslant t_{0}\end{cases}$

where:

$\mathrm{F}_{\min }=$ initial fluorescence, dependent on pseudofluorescence;

$\mathrm{F}_{\mathrm{dt}}=$ maximal fluorescence increase, dependent on the amount of blood with fluorescein, blocking of pigments, etc;

$\mathrm{t}_{0}=$ the onset of the filling process, reflecting the delay time between injection site, and the eye;

$\tau=$ tau, the time constant of the model.

The model curve is shown in Figure 1. Normally, $F_{\min }$ is low and may depend on the colour filters used; the fluorescence factor of interest is $F_{\mathrm{dt}}$.

The description of the pattern of each dye curve using the aforementioned four variables was found to be accurate. Accuracy of curve fitting is usually defined as the difference between the model curve and the actual dye build up curve. This simple model describes approximately $90 \%$ of dye curve information. There is a small though statistically significant improvement in fit by the introduction of an extra time constant in the model. ${ }^{33}$ The analysis using this mathematical model enables not only a description of the dye curves but also a possible of interpretation in terms of rheological relevant variables, using a rheological one compartmental model. This model describes the filling of the choriocapillaris, the compartment, with dye during fluorescein angiography. Translating the descriptive variables into rheological variables, $t_{0}$ reflects the delay time between the moment of injection and appear- ance in the posterior pole, introduced by the circulation between the site of injection and the eye, and $\tau$ can be regarded as the local blood refreshment time. Using fluorescein angiography only fluorescence from superficial layers up to and including the choriocapillaris, excluding the large choroidal vessels, will contribute to the background fluorescence. The dye curves, not located on retinal vessels, will reflect the fluorescence of the choriocapillaris. The blood refreshment time $\tau$ describes the time needed to replace the blood volume in a certain tissue, in this case the choriocapillaris. Consequently, if blood flow is decreased, the time needed to replace the blood volume will be longer, and the blood refreshment time will increase. In an animal experiment it was demonstrated that the angiographically derived $\tau$ values are indeed correlated negatively with blood flow of the peripapillary choroid, determined by the radioactively labelled microspheres method. The latter can be regarded as a standard method in experimental blood flow measurements, and enables absolute flow measurements in separate ocular tissues-for example, the peripapillary choroid. In comparing the results of the two methods, $\tau$ was to be interpreted as the local blood refreshment time of the peripapillary choroid, and another rheological variable, the local blood volume of the choriocapillaris, could be estimated. ${ }^{34}$

The goal of this study was to evaluate whether choroidal haemodynamics, parametrised using this method based on fluorescein angiograms, are altered in patients with POAG, NPG, or ocular hypertension compared with normal healthy subjects.

\section{Patients and methods}

In this study 106 glaucoma and ocular hypertension (OHT) patients from the outpatient clinic of the Glaucoma Center of the Academic Medical Center participated. Twenty two accompanying people and staff members were asked to participate as normal, healthy subjects.

Cases of lenticular or corneal disease (precluding fluorescein angiography), diabetes mellitus, severe cardiovascular disorders, history of allergy, or known allergy to fluorescein or filtering surgery were excluded. If both eyes met the aforementioned criteria, the study eye was selected randomly.

Inclusion criteria for normal subjects were no history of ocular diseases, normal findings of the slit lamp examination, Goldmann tonometry (IOP $\leqslant 18 \mathrm{~mm} \mathrm{Hg}$ ), ophthalmoscopy, and nerve fibre evaluation.

Patients were included with the diagnosis OHT, POAG, or NPG, in case of open angles examined by gonioscopy, glaucomatous cupping of the disc, and glaucomatous visual field defects (using the Humphrey 30-2 program). Glaucoma patients were diagnosed as POAG (48 patients) if the mean IOP derived from diurnal IOP measurements (off treatment) was greater than $21 \mathrm{~mm} \mathrm{Hg}$. The diagnosis of NPG (46 patients) was based on the same criteria, with the exception of a statistically normal IOP, in this case $\leqslant 21 \mathrm{~mm} \mathrm{Hg}$. To allow for 


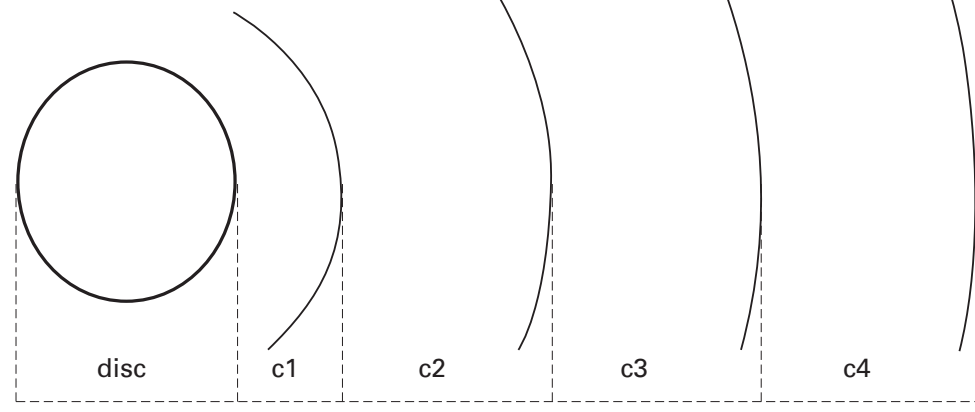

Figure 2 Schematic drawing of the circular areas centred around the disc, used to divide the posterior pole into areas defined as fractions of disc diameters.

measurement errors, an isolated intraocular pressure up to $26 \mathrm{~mm} \mathrm{Hg}$ in a diurnal intraocular pressure curve was accepted. $\mathrm{Pa}-$ tients were diagnosed as OHT in the absence of indications of glaucomatous damage (no glaucomatous cupping, normal retinal nerve fibre layers, and no visual field defects) with a mean IOP derived from a diurnal IOP curve greater than $21 \mathrm{~mm} \mathrm{Hg}$.

After the angiogram the IOP and blood pressure were measured. The average ocular perfusion pressure was defined as:

perfusion pressure $=$

$$
\mathrm{p}_{\text {diastolic }}+1 / 3\left(\mathrm{p}_{\text {systolic }}-\mathrm{p}_{\text {diastolic }}\right)-\text { IOP }
$$

The study was approved by the medical ethics committee of the Academic Medical Center and informed consent was obtained from all participants.

In order to induce mydriasis, tropicamide $0.5 \%$ was administered in the study eye. After proper focusing of the scanning laser ophthalmoscope (SLO, Rodenstock, fixed gain, 160 $\mu \mathrm{W}$ blue argon laser, $40^{\circ}$ field) $1.5 \mathrm{ml}$ sodium fluorescein $25 \%$ was injected in the antecubital vein (catheterised with 21 gauge butterfly needle). The fluorescein angiograms were recorded on superVHS video tape (Panasonic AG-7350). The pictures were centred on the disc.

The angiogram was digitised into 100 pictures. The spatial resolution was $380 \times 228$ pixels; the temporal resolution was one picture per second just after the injection, gradually increasing to a maximum of five pictures per second during the arterial phase, and decreasing to one picture per second in the venous phase. The pictures were aligned by a specially made automatic procedure using cross correlation of pictures, analogous to methods used in radiology for image alignment. ${ }^{35}$ For the central $228 \times 228$ pixels the model analysis was performed.

For each of the $228 \times 228$ pixels a dye curve was constructed. Fluorescence intensity was plotted on the ordinate, time was plotted on the abscissa. Every dye curve was analysed according to the previously mentioned model, equation (1).$^{32}$ Hence, for each location of the posterior pole haemodynamics are parametrised, described by the aforementioned variables.

To study the spatial distribution of the model variables the location of the disc was manually indicated with the help of specially made software on a digitised red free SLO picture, matched with the aligned angiogram series. Subsequently, circular areas centred around the disc could be analysed. As shown in Figure 2, in this way the posterior pole of each patient was divided into a circle covering the disc, and circular regions extending from 1 to 1.5 disc diameters and subsequent 1.5-2.5, 2.5-3.5, and 3.5-4.5 disc diameters from the centre of the disc. Pixels overlying larger retinal arteries and veins up to the second order branches were automatically detected, without need of human input, by an automated image analysis algorithm implemented on a personal computer. They were excluded from further analysis. Generally, approximately 6000 pixels $(11.5 \%)$ were excluded from further analysis. For each region the mean and standard deviation of the model variables were calculated.

Since the variables in the study population did not follow normal distribution, nonparametric statistical tests were used. To examine the differences in model variables between groups of diagnosis, the Kruskal-Wallis analysis of variance (ANOVA) by ranks and the Mann-Whitney U test (MWU) were used. The Kruskal-Wallis ANOVA can be regarded as the non-parametric equivalent of the analysis of variance with multiple groups; the MannWhitney $U$ test can be regarded as the non-parametric equivalent of the $t$ test for two independent samples. To investigate the relation between fundus location and variable values, Friedman's two way ANOVA, the nonparametric equivalent of repeated measures ANOVA, was used. With regard to correlations the Spearman rank order correlation was used.

\section{Results}

In Table 1 some characteristics of the groups are given: sex, age, a visual field index (corrected pattern standard deviation, CPSD), mean intraocular pressure (IOP), mean arterial pressure, perfusion pressure, smoking, and use of topical medication. The intraocular pressure is the average of the readings from the diurnal IOP curve; the arterial pressure and perfusion pressure are based on the measurements just after angiography. Males and females were equally distributed over the groups. All POAG patients received topical treatment, mostly topical $\beta$ blockers. Only 20 of the 46 NPG patients received topical treatment. As follows from the diagnostic criteria, statistical differences in the intraocular pressure were seen among the study groups (KW ANOVA $\mathrm{p}<0.001)$. The highest IOP values were found in the POAG patients. The ocular perfusion pressure did not differ among the groups (KW ANOVA $p=0.14$ ); the lowest perfusion pressure was found in the POAG group. The arterial diastolic and systolic pressures were not statistically different among the groups (KW ANOVA $\mathrm{p}=0.09)$. The average disc areas (SD) 
Table 1 Number, sex, age (SD), corrected pattern standard deviation (CPSD, (SD)), intraocular pressure (SD), arterial systolic and diastolic blood pressure (SD), perfusion pressure (SD), smoking habits, and topical therapies of the patients

\begin{tabular}{|c|c|c|c|c|}
\hline & Normals & $O H T$ & $P O A G$ & $N P G$ \\
\hline No & 22 & 12 & 48 & 46 \\
\hline $\operatorname{Sex}(M / F)$ & $15 / 12$ & $7 / 5$ & $23 / 25$ & $24 / 22$ \\
\hline Age (years) & $52.4(15.2)$ & $59.0(8.6)$ & $64.2(10.8)$ & $68.8(10.9)$ \\
\hline CPSD (dB) & $3.4(3.1)$ & $2.4(2.5)$ & $9.4(3.6)$ & $9.5(3.1)$ \\
\hline $\mathrm{IOP}(\mathrm{mm} \mathrm{Hg})$ & $13.7(2.3)$ & $26.5(6.1)$ & $30.4(11.3)$ & $18.1(2.7)$ \\
\hline Systolic BP (mm Hg) & $140.2(11.5)$ & $142.8(13.0)$ & $146.5(15.9)$ & $145.6(12.8)$ \\
\hline Diastolic BP (mm Hg) & $75.9(9.4)$ & $84.2(11.5)$ & $85.2(13.0)$ & $83.6(11.9)$ \\
\hline Ocular PP (mm Hg) & $83.4(9.1)$ & $76.8(15.4)$ & $77.7(17.9)$ & $86.2(11.9)$ \\
\hline Smokers & 6 & 4 & 12 & 14 \\
\hline No topical medication & 22 & 5 & 0 & 26 \\
\hline Non-selective $\beta$ blocker & 0 & 4 & 40 & 14 \\
\hline Selective $\beta 1$ blocker & 0 & 0 & 5 & 5 \\
\hline Pilocarpine & 0 & 2 & 12 & 1 \\
\hline Adrenaline & 0 & 3 & 6 & 1 \\
\hline Guanethidine & 0 & 0 & 3 & 0 \\
\hline
\end{tabular}

in the controls, OHT, POAG, and NPG patients were $2.19(0.42), 2.33$ (0.73), 2.30 (0.56), and $2.10(0.45) \mathrm{mm}^{2}$ respectively; these areas were not statistically different among the study groups (KW ANOVA $\mathrm{p}=0.24)$.

Kruskal-Wallis ANOVA with or without adjustment for age showed that the differences between the groups could not be attributed to differences in age between the groups. As to the adjustment for age, all subjects (controls and patients) were used. Moreover, there appeared to be no correlation between age and the model parameters. Because of the relatively large spread a non-linear relation between age and model parameters was not considered, and age influences were not taken into account.

The median values for the three model parameters for the five fundus locations are given in Table 2. The $\mathrm{p}$ value, given for each parameter, is the outcome of the KruskalWallis ANOVA, and indicates whether some significant difference in the median variable values in the four study groups exists. The model parameter maximal fluorescence $\left(F_{d t}\right)$ and the time constant of the model, $\tau$, show such a difference. The time of first dye appearance $\left(\mathrm{t}_{0}\right)$ did not show a statistically significant difference. Since multiple ANOVAs are performed, the significance of differences with borderline $p$ values, such as are found in the choroidal maximal fluorescence $\left(\mathrm{F}_{\mathrm{dt}}\right)$ variable values will have to be considered with care. The $\mathrm{p}$ values for the variable $\tau$ were much smaller.

In Figure 3 the 'standardised' averages (25th-75th percentile) of the variable values for the patient groups and fundus locations are given. These values were standardised using the individual mean of the disc and choroidal values. Friedman ANOVA showed that for all three variables there exists a significant relation between location and value $(\mathrm{p}<0.001)$. It must be noted that the generally large spread around the medians from Table 2 is caused by interindividual differences, as the spread is much smaller in Figure 3. In the variables $F_{d t}$ and $t_{0}$, in particular, an evident dependence on the location exists. With the exception of the $\mathrm{F}_{\mathrm{dt}}$ of the disc, no statistically significant differences in this location dependence could be demonstrated.

The variable $\mathrm{F}_{\mathrm{dt}}$ shows the largest significant differences between the patient groups with respect to the area of the disc. Whereas the fluorescence of the normals and OHT patient was comparable, the disc fluorescence in the POAG and NPG groups was significantly lower compared with normals (MWU, $\mathrm{p}<0.01)$. However, the choroidal fluorescence was significantly lower only in the POAG group (MWU, $\mathrm{p}<0.02$ ).

Table 2 Median values (25th percentile-75th percentile) of the three model parameters in the study groups for each fundus location. The $p$ values under the variable values indicate the outcome of the Kruskal-Wallis ANOVA showed that only the variables $F_{d t}$ and $\tau$ were significantly different among the study groups. Friedman ANOVA showed significant relations between location and variable values

\begin{tabular}{|c|c|c|c|c|c|}
\hline & $F_{d t} d i s c$ & $F_{d t} c 1$ & $F_{d t} c 2$ & $F_{d t} c 3$ & $F_{d t} c 4$ \\
\hline $\mathrm{N}$ & $66.4(51.0-116.8)$ & $58.1(45.6-88.2)$ & $55.9(41.5-77.2)$ & $55.0(41.2-74.5)$ & $53.4(37.8-69.2)$ \\
\hline OHT & $62.2(48.6-84.2)$ & $57.5(40.3-73.2)$ & $56.0(40.0-76.3)$ & $52.0(34.1-67.6)$ & $48.3(33.4-66.4)$ \\
\hline POAG & $37.0(28.2-54.1)$ & $34.5(25.7-58.8)$ & $33.3(24.2-51.9)$ & $29.6(21.1-46.7)$ & $29.3(21.4-45.1)$ \\
\hline \multirow[t]{3}{*}{ NPG } & $48.1(29.5-68.8)$ & $44.1(27.0-65.0)$ & $42.1(27.1-69.2)$ & $40.4(24.7-67.0)$ & $40.0(23.7-65.2)$ \\
\hline & $\mathrm{p}=0.0003$ & $\mathrm{p}=0.024$ & $\mathrm{p}=0.035$ & $\mathrm{p}=0.041$ & $\mathrm{p}=0.018$ \\
\hline & $t_{0}$ disc & $t_{0} c 1$ & $t_{0} c 2$ & $t_{0} c 3$ & $t_{0} c 4$ \\
\hline $\mathrm{N}$ & $14.1(12.1-14.7)$ & $14.4(12.4-15.0)$ & $14.6(12.2-15.3)$ & $14.6(12.4-15.3)$ & $14.7(12.7-15.2)$ \\
\hline OHT & $15.1(13.7-19.0)$ & $15.1(13.9-19.3)$ & $15.7(14.4-19.8)$ & $16.3(14.4-20.1)$ & $16.5(14.1-20.1)$ \\
\hline POAG & $15.1(13.9-20.9)$ & $14.9(13.2-20.6)$ & $15.1(13.8-20.7)$ & $15.3(13.2-20.6)$ & $15.4(12.6-20.1)$ \\
\hline \multirow[t]{3}{*}{ NPG } & $15.5(14.0-19.5)$ & $16.1(13.7-20.0)$ & $15.8(13.5-20.2)$ & $16.0(13.3-20.1)$ & $16.2(12.8-20.3)$ \\
\hline & ns & ns & ns & ns & ns \\
\hline & $\tau$ disc & $\tau c 1$ & $\tau c 2$ & $\tau c 3$ & $\tau c 4$ \\
\hline $\mathrm{N}$ & $3.8(3.3-4.4)$ & $4.1(3.5-4.5)$ & $4.1(3.6-4.6)$ & $4.2(3.7-4.6)$ & $4.2(3.8-4.8)$ \\
\hline $\mathrm{OHT}$ & $4.1(3.0-5.0)$ & $4.4(3.4-6.3)$ & $4.5(3.8-6.2)$ & $4.5(3.8-6.2)$ & $4.5(4.0-6.2)$ \\
\hline POAG & $5.0(4.1-6.0)$ & $5.5(4.3-6.8)$ & $5.6(4.4-6.7)$ & $5.7(4.4-7.0)$ & $5.7(4.5-7.3)$ \\
\hline \multirow[t]{2}{*}{ NPG } & $6.6(4.8-7.9)$ & $7.5(5.5-9.3)$ & $6.9(5.4-9.4)$ & $7.0(5.5-9.4)$ & $7.0(5.6-9.4)$ \\
\hline & $\mathrm{p}<0.0001$ & $\mathrm{p}<0.0001$ & $\mathrm{p}<0.0001$ & $\mathrm{p}<0.0001$ & $\mathrm{p}<0.0001$ \\
\hline
\end{tabular}



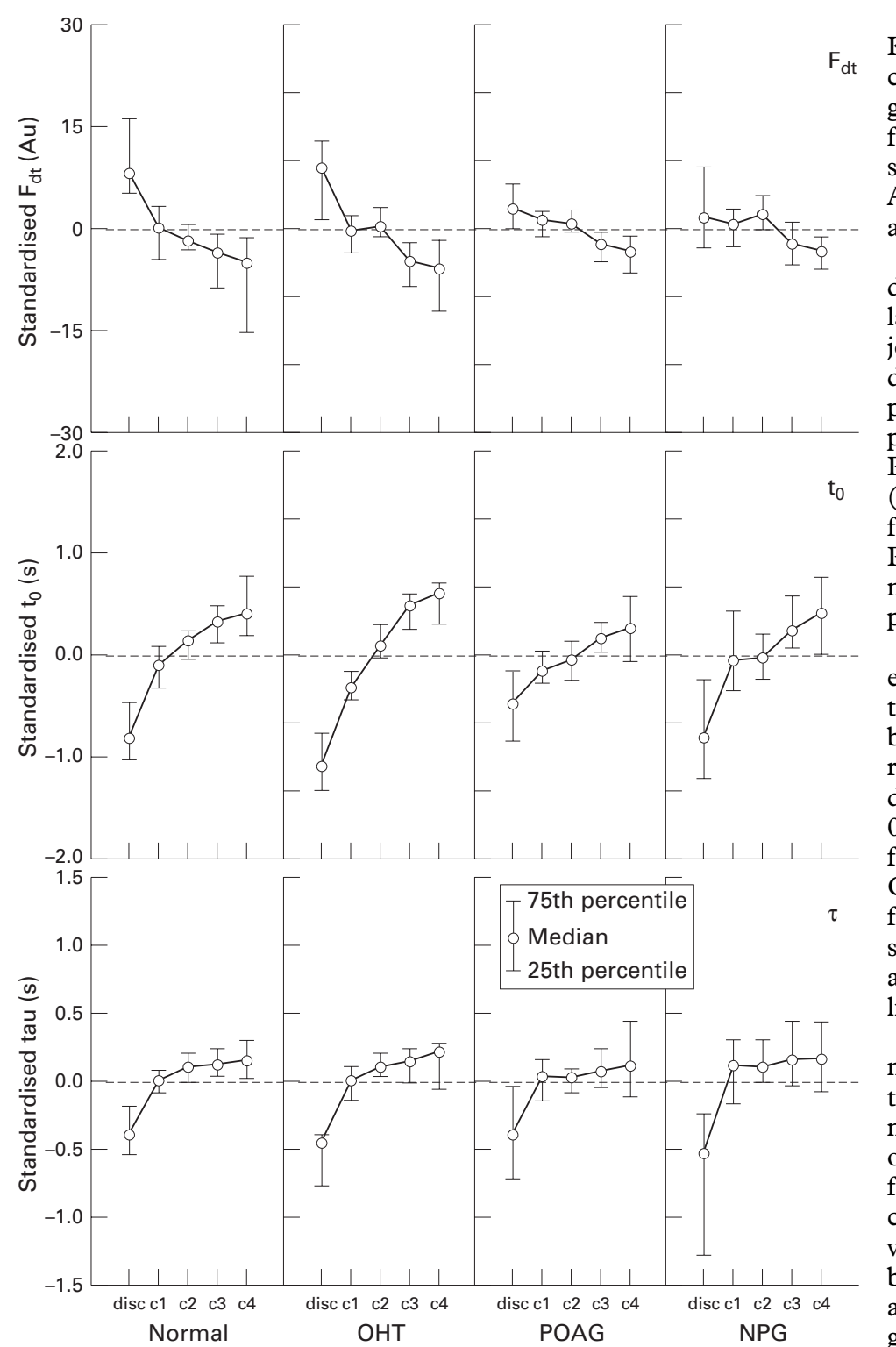

Figure 3 Standardised median parameter values and 25th-75th percentile plotted as a function of eccentricity for the four study groups (OHT, ocular hypertension; $P O A G$, primary open angle glaucoma; $N P G$, normal pressure glaucoma). $F_{d t}=$ maximum fluorescence, $t_{0}=$ first appearance of dye, $\tau=$ the time constant of the model. There exists a significant relation between parameter value and eccentricity in all cases.

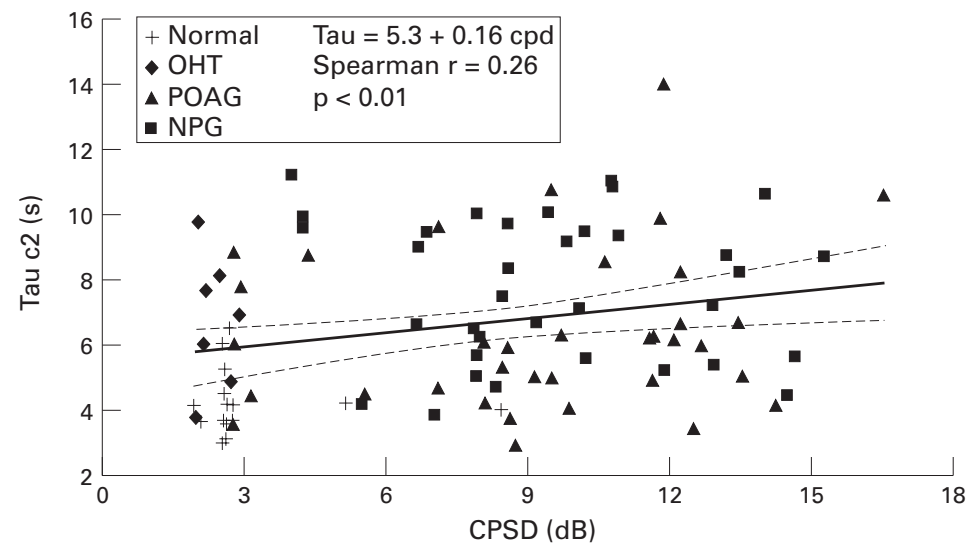

Figure 4 Mean $\tau$ values (seconds) for a choroidal area (c2) plotted against corrected pattern standard deviation (CPSD) (dB), showing a weak correlation (Pearson's $r=0.27$, $p<0.01$ ). Note that if the visual field data from the normals are omitted, the relation loses statistical significance.
The time of first fluorescence, $t_{0}$, using Kruskal-Wallis ANOVA did not show statistically significant differences among these groups. The analysis of the relation between fundus location and onset of fluorescence showed a very significant relation (Friedman ANOVA, $\mathrm{p}<0.001)$. More eccentric locations are associated with later $t_{0}$.

The model parameter $\tau$ showed the strongest differences between the patient groups. The largest difference existed between normal subjects and NPG patients (MWU, $p<0.001$ ). The difference between normal subjects and POAG patients was significant as well (MWU, $\mathrm{p}<0.001)$. Finally, the differences between the POAG and NPG patients were also significant (MWU, $\mathrm{p}<0.01$ ). The slowest tau values were found in the NPG patients, followed by the POAG patients. The difference between normal subjects and ocular hypertensives did not prove to be statistically significant.

The correlation between visual field defect, expressed as corrected pattern standard deviation (CPSD), and model parameter $\tau$ proved to be weak though significant. The Spearman rank order correlation between (a) $\tau$ values for disc and choroid and (b) CPSD ranged from 0.26 to $0.36(p<0.02)$. In Figure 4 the $\tau$ values for the choroid area 2 are plotted against the CPSD values. However, if the visual field data from normal subjects were left out, no significant relation between model parameters and visual field parameters could be established.

The influence of topical antiglaucomatous medication, smoking, perfusion pressure, intraocular pressure, and arterial pressure on the model parameters was investigated by analysis of variance after adjustment for that specific factor - for example, topical medication. In the case of antiglaucomatous medication, the values for the model parameters were adjusted by subtracting the difference between overall average and the average of the medication group from the value of the individual. Adjustment for perfusion pressure, arterial pressures, and IOP were based on the regression between model parameters and studied pressure. The Kruskal-Wallis ANOVA of the adjusted variables showed that the differences in haemodynamic variables between the groups cannot be attributed to differences in, for example, use of antiglaucomatous medication or perfusion pressure. Moreover, there existed no statistically significant correlation between haemodynamic model parameter on the one hand and IOP, arterial pressure or perfusion pressure on the other.

\section{Discussion}

Through parametrisation of dye build up curves derived from video fluorescein angiograms, differences in both choroidal and disc haemodynamics have been found between healthy subjects and glaucoma patients, as well as between glaucomatous subpopulations. In particular, the time constant of the model, $\tau$, theoretically reflecting the choroidal blood refreshment time, showed large differences 
between normals and glaucoma patients, as well as between primary open angle and normal pressure glaucoma patients.

The analysis of dye build up curves using an exponential model not only enables a mere mathematical description, but also offers clinically useful, quantitative haemodynamic variables. Confining oneself to choroidal dye curves, the exponential, descriptive variable can be theoretically interpreted as variables of a rheological one compartmental model. The variable $t_{0}$ reflects the circulation between antecubital vein and posterior pole; $\mathrm{F}_{\mathrm{dt}}$, among other things, reflects the amount of blood present, and $\tau$ can be regarded as the local choroidal blood refreshment time. Only fluorescence from more superficial layers up to and including the choriocapillaris, excluding fluorescence from larger choroidal vessels (as in indocyanine green angiography), contributes to the dye curve. ${ }^{32}$ Therefore, the blood refreshment time $\tau$ reflects the time needed to replace the blood volume in a certain tissue volume, in this case the choriocapillaris.

This interpretation in terms of a one compartmental model only applies to choroidal dye curves. As in all model analyses reality is simplified, yet it is obvious that the choriocapillaris, a clearly defined monolayer of large capillaries, can be regarded as a volume. One of the complicating factors might be the fact that fluorescein extravasates out of the fenestrated capillaries.

Since the vasculature of the optic nerve head is much more complicated and in glaucomatous disease tissue loss occurs, an extrapolation of descriptive model parameters towards haemodynamic relevant variables is much more difficult.

Other authors have also found haemodynamic differences between normals and glaucoma patients; retinal dye transit times are prolonged in POAG patients, ${ }^{17}$ ophthalmic artery velocities measured with duplex ultrasound are reduced..$^{76}$ In glaucoma patients qualitative changes of the choroidal circulation have also been found. $.^{1-3} 28-30$

Differences in the fluorescence of the disc have also been found. Using image analysis of photographic fluorescein angiograms an increased number of filling defects and a larger area of disc filling defects has been found in ocular hypertensives and progressive ocular hypertensives. ${ }^{37-39}$ Hayreh found that in glaucoma patients fluorescence of the disc and choroid are about the same, whereas fluorescence of the disc in normal subjects is much higher. ${ }^{140}$ These findings are in agreement with our findings that the maximal fluorescence of the disc is significantly lower in POAG and NPG and that the disc fluorescence in normal subjects is higher than that of the choroid.

The vasculature of the disc has an intricate three dimensional structure. Several layers with presumably different arterial blood supplies can be distinguished. In pathological states tissue loss occurs, which makes the interpretation of angiographical findings of the disc even more difficult.
Another complicating factor influencing the magnitude of $F_{d t}$ is the influence of pigments. Apart from differences in illumination and opacities of ocular media, differences in, for example, pigment epithelium may play their role. ${ }^{41}$ Another factor is that $\mathrm{F}_{\mathrm{dt}}$ is dependent on the amount of blood and fluorescein. One could speculate that the small difference in choroidal fluorescence between normal subjects and glaucoma patients may be due to a thinner choriocapillaris. However, the variable $\mathrm{F}_{\mathrm{dt}}$ shows large variation and there is considerable overlap between normal values and glaucomatous values and the magnitude of $F_{d t}$ is influenced by a multitude of variables.

The variable $t_{0}$ did not differ statistically significantly between the four subgroups, although, for the glaucomatous groups, there was a tendency towards longer $t_{0}$ values. With larger populations this tendency might reach statistical significance. The magnitude of $t_{0}$ is in agreement with previous findings using human observer dependent methods. ${ }^{30}$ This variable describes the circulation time between site of injection, the antecubital vein, and the posterior pole. Thus, differences in many parts of the circulation, such as heart and lungs, but also carotid, ophthalmic and ciliary arteries may influence $t_{0}$. To delineate these factors and influences the use of other techniques such as colour Doppler ultrasound imaging may offer more insight. Through the combination of angiography and scintigraphy a prolonged carotis-retina time has been found in NPG patients compared with normal subjects. The subclavian-carotis time and the retinal passage time did not differ. ${ }^{42}$ Thus, there may exist local differences in the circulation between antecubital vein and posterior pole, and especially in the circulation of the ophthalmic and central retinal artery.

There appeared to be a very significant relation between the magnitude of $t_{0}$ and fundus locations. Generally, later $t_{0}$ values are found at more eccentric locations. This phenomenon may be caused by propagation of the dye front in the larger choroidal vessels which enter the globe near the disc. However, this is speculative and will be discussed in a separate paper.

The local choroidal blood refreshment time $\tau$ was significantly longer in POAG and NPG patients. Others have also found altered peripapillary choroidal haemodynamics. ${ }^{28-30}$ However, these studies did not use automated analysis techniques, but relied on the eye of the observer and are more or less qualitative in character. Furthermore, image analysis as used in our study not only enables a standardised description of the angiograms, but also the extraction of variables, such as the local blood refreshment time $\tau$. This variable cannot be quantified from an angiogram by eye, and offers a new possibility to study choroidal haemodynamics.

Another quality of the model parameter $\tau$ is that it does not depend on confounding factors like fluorescence intensities. By means of the exponential analysis, differences in fluorescence intensities are accounted for through the variable $\mathrm{F}_{\mathrm{dt}}$. Most qualitative methods and 
methods measuring ascending slopes of dye curves are intrinsically dependent on the amount of fluorescence. The slope is generally calculated as $\Delta$ fluorescence/ $\Delta$ time. Therefore, $\tau$ values cannot be compared with variables like slopes of dye curves, as used in previous studies. ${ }^{37}$

Since choroidal blood refreshment times were significantly longer in glaucoma patients, and especially in normal pressure glaucoma patients, it may be concluded that the choroidal circulation is slowed down in glaucoma patients. We will discuss some factors which possibly are the cause of these differences.

First of all, the analysis, using the annular regions (with the disc as a standard), may be biased. Some studies showed that the size of the disc is somewhat larger in NPG patients compared with POAG and normal controls. ${ }^{43}{ }^{44}$ If there is a difference, this would imply that, compared with POAG patients, slightly more eccentric locations (approximately $7 \%$ of the disc diameter) are measured in NPG patients; this is negligible compared with the large intergroup differences in blood refreshment time $\tau$. However, as reported by others, ${ }^{45}$ a difference in disc size between the groups could not be demonstrated. Therefore, systematic differences induced by disc sizes are rather unlikely.

Since the normal subjects are on average slightly younger and possibly healthier, perhaps the differences in variable values between the study groups reflect differences in their vascular system. However, statistical analysis did not reveal age influence on the variables. Furthermore, the two glaucomatous groups, POAG and NPG, cover the same age and visual field defect range, and, comparing these two groups, $\tau$ values were significantly longer in the NPG patients compared with POAG patients. Thus, it seems unlikely that age had a confounding influence.

Another factor may be the influence of topical medication. The groups are clearly unmatched for the use of topical antiglaucomatous therapy. Medication may influence blood flow in the posterior pole either via a perfusion pressure effect or via direct action on blood flow; $\beta$ blockers especially may influence ocular blood flow. However, in the current study differences in topical medication and smoking could not explain the differences in the blood flow variables; on the other hand, this study was not designed for the evaluation of haemodynamic effects of medication, and one has to be very careful with the interpretation of the result regarding medication.

Another difference between the groups is perfusion pressure-the difference between average arterial blood pressure and intraocular pressure. The perfusion pressure influences ocular blood flow. From a comparative study in rabbits it appears that at least, in the rabbit, perfusion pressure to a large extent influences choroidal haemodynamics. ${ }^{34}$ However, in this animal experimental study the perfusion pressure was changed artificially over a large range of pressures. The physiological perfusion pressure range is much smaller in humans, thus possibly obscuring a relation between perfusion pressure or intraocular pressure and choroidal haemodynamics. Subsequently, in the present study there appeared to be no relation between blood refreshment time on the one hand and perfusion, intraocular, or arterial pressure on the other. Furthermore, also from the difference between the haemodynamics in POAG and NPG patients, one may conclude that the prolonged choroidal blood refreshment time in NPG patients is not related to differences in intraocular pressure, mean arterial pressure, or ocular perfusion pressure.

The average intraocular pressure in POAG patients was much higher than in NPG patients (Table 1). If differences in intraocular pressure and, indirectly, perfusion pressure would have influenced choroidal haemodynamics, one would expect reduced blood flow, especially in patients with high intraocular pressures and lower perfusion pressures. However, although somewhat slower $\tau$ values have been found in the POAG group, the slowest $\tau$ values have been found in the NPG group. It may be theorised that the changes in some POAG patients, with high intraocular pressures, reflect a perfusion pressure effect. However, in the case of the NPG patients some local change of the vasculature in the posterior pole may explain the slow choroidal haemodynamics.

Because of the common source of blood supply via the short posterior ciliary arteries of large parts of the optic nerve head and the peripapillary choroid one might expect a relation between blood flow in the optic nerve head and choroidal blood flow.

The analysis of choroidal dye build up curves may be a clinically useful method for the study of haemodynamics in glaucoma patients. Clinicians have a long standing experience regarding the method of fluorescein angiography. The scanning laser ophthalmoscope and image analysis have made quantitative measures possible and provided a 'new' variable, which cannot be measured through the clinician's eyes. Haemodynamic abnormalities have been found in a sizeable number of glaucoma patients and particularly normal pressure glaucoma patients.

Part of this study has been presented at the annual meeting of the Association for Research in Vision and Ophthalmology, 1994 .

1 Hayreh SS, Walker WM. Fluorescent fundus photography in glaucoma. Am f Ophthalmol 1967;63:982-9.

2 Hitchings RA, Spaeth GL. Fluorescein angiography in chronic simple and low-tension glaucoma. Brf Ophthalmol 1977;61:126-32.

3 Spaeth GL. The pathogenesis of nerve damage in glaucoma: contributions of fluorescein angiography. New York: Grune \& Stratton, 1977.

$4 \mathrm{Alm}$ A, Bill A. The oxygen supply to the retina. II Effects of high intraocular pressure and of increased arterial carbon dioxide tension on uveal and retinal blood flow in cats. A study with radioactively labelled microspheres including flow determinations in brain and some other tissues. Acta Physiol Scand 1972;84:306-19.

5 Tamaki Y, Araie M, Kawamoto E, Eguchi S, Fujii H. Noncontact, two-dimensional measurement of retinal microcirculation using laser speckle phenomenon. Invest Ophthalculation using laser speckle

6 Riva CE, Petrig B. Blue field entoptic phenomenon and Riva CE, Petrig B. Blue field entoptic phenomenon and
blood velocity in the retinal capillaries. F Opt Soc Am 1980; 70:1234-8. 
7 Rojanapongpun P, Drance SM, Morrison BJ. Ophthalmic artery flow velocity in glaucomatous and normal subjects. Brf Ophthalmol 1993;77:25-9.

8 Riva CE, Grunwald JE, Sinclair SH, Petrig BL. Blood velocity and volumetric flow rate in human retinal vessels. Invest Ophthalmol Vis Sci 1985;26:1124-32.

9 Riva CE, Grunwald JE, Sinclair SH. Laser Doppler measurement of relative blood velocity in the human optic nerve head. Invest Ophthalmol Vis Sci 1982;22:241-8.

10 Riva CE, Cranstoun SD, Grunwald JE, Petrig BL. Choroidal blood flow in the foveal region of the human ocular fundus. Invest Ophthalmol Vis Sci 1994;35:4273-81.

11 Michelson G, Schmauss B. Two dimensional mapping of the perfusion of the retina and optic nerve head. $\mathrm{BrF} \mathrm{Oph-}$ thalmol $1995 ; 79: 1126-32$

12 Holló G, Van den Berg TJTP, Greve EL. Scanning laser Doppler flowmetry in glaucoma. Submitted for publication.

13 Hickham JB, Frayser R. A photographic method for measuring the mean retinal circulation time using fluoresmeasuring the mean retinal circulation

14 Bulpitt CJ, Dollery CT. Estimation of retinal blood flow by measurement of the mean circulation time. Cardiovasc Res 1971:5:406-12.

15 Riva CE, Feke GT, Ben-Sira I. Fluorescein dye-dilution technique and retinal circulation. Am $\mathcal{F}$ Physiol 1978;234 H315-22.

16 Wolf S, Jung F, Kiesewetter H, Körber N, Reim M. Video fluorescein angiography: method and clinical application. Graefes Arch Clin Exp Ophthalmol 1989;227:145-51.

17 Wolf S, Arend O, Sponsel WE, Schulte K, Cantor LB, Reim M. Retinal hemodynamics using scanning laser ophthalmoscopy and hemorheology in chronic open-angle glaucoma. Ophthalmology 1993;100:1561-6.

18 Wolf S, Arend O, Reim M. Measurement of retinal hemodynamics with scanning laser ophthalmoscopy: reference values and variation. Surv Ophthalmol 1994;38(suppl):S95100 .

19 Flower RW, Klein GJ. Pulsatile flow in the choroida circulation: a preliminary investigation. Eye 1990;4:310-8.

20 Klein GJ, Baumgartner RH, Flower RW. An image processing approach to characterizing choroidal blood flow. Invest ing approach to characterizing choro

21 Flower RW. Extraction of choriocapillaris hemodynamic data from ICG fluorescence angiograms. Invest Ophthalmol Vis Sci 1993;34:2720-9.

22 Prünte C, Niesel P. Quantification of choroidal blood-flow parameters using indocyanine green video-fluorescence angiography and statistical picture analysis. Graefes Arch Clin Exp Ophthalmol 1988;226:55-8.

23 Hyvarinen L, Maumenee AE, George T, Weinstein GW. Fluorescein angiography of the choriocapillaris. Am f Ophthalmol 1969;67:653-66.

24 Oosterhuis JA, Boen-Tan TN. Choroidal fluorescence in the normal human eye. Ophthalmologica 1971;162:246-60.

25 Shimizu K, Yokochi K, Okano T. Fluorescein angiography of the choroid. Fpn $\mathcal{f}$ Ophthalmol 1974;18:97-108.

26 Hayreh SS. The choriocapillaris. Albrecht Von Graefes Arch Klin Exp Ophthalmol 1974;192:165-79.

27 Hayreh SS. In vivo choroidal circulation and its watershed zones. Eye 1990;4:273-89.

28 Raitta C, Sarmela T. Fluorescein angiography of the optic disc and the peripapillary area in chronic glaucoma. Acto Ophthalmol (Copenh) 1970;48:303-8.
29 Laatikainen L. Fluorescein angiographic studies of the peripapillary and perilimbal regions in simple, capsular and ow-tension glaucoma. Acta Ophthalmol (suppl) 1971;111: $3-83$.

30 Geijssen HC. Studies on normal pressure glaucoma. Amstelveen: Kugler Publications, 1991.

31 Lambrou GN, Van den Berg TJTP, Greve EL. Vascular plerometry of the choroid. An approach to the quantificaion of choroidal blood flow using computer-assis pron Greve EL, eds. Ocular blood flow in glaucoma. Amstelveen: Kugler \& Ghedeni, 1989:287-94.

32 Lambrou GN. Choroidal plerometry: a method for evaluating the choroidal blood supply in clinical practice. Kugler Publications: Amsterdam, 1993.

33 VanStokkum IHM, Lambrou GN, Van den Berg TJTP. Hemodynamic parameter estimation from ocular fluorescein angiograms. Graefes Arch Clin Exp Ophthalmol 1995;233:123-30.

34 Duijm HFA, Rulo AHF, Astin M, Mäepea O, Van den Berg TJTP, Greve EL. Study of choroidal blood flow by comparison of SLO fluorescein angiography and microspheres. Exp Eye Res 1996;63:693-704.

35 Appledorn CR, Oppenheim BE, Wellman HN. An automated method for the alignment of image pairs. 7 Nucl Med $1980 ; 21: 165-7$.

36 Harris A, Sergott RC, Spaeth GL, Katz JL, Shoemaker JA, Martin BJ. Color Doppler analysis of ocular vessel blood velocity in normal-tension glaucoma. Am $\mathcal{f}$ Ophthalmol 1994;118:642-9.

37 Tuulonen A, Nagin P, Schwartz B, Wu DC. Increase of pallor and fluorescein-filling defects of the optic disc in the follow-up of ocular hypertensives measured by computerized image analysis. Ophthalmology 1987;94:558-63.

38 Loebl M, Schwartz B. Fluorescein angiographic defects of the optic disc in ocular hypertension. Arch Ophthalmol 1977;95:1980-4.

39 Schwartz B, Rieser JC, Fishbein SL. Fluorescein angiographic defects of the optic disc in glaucoma. Arch Ophthalmol 1977;95:1961-74.

40 Hayreh SS. Blood supply of the optic nerve head and its role in optic atrophy, glaucoma, and oedema of the optic disc. Br F Ophthalmol 1969;53:721-48.

41 Geeraets WJ, Williams RC, Chan G, Ham WT, Guerry DP, Schmidt FH. The relative absorption of thermal energy in retina and choroid. Invest Ophthalmol 1962;1:340-7.

42 Nasemann JE, Carl T, Pamer S, Scheider A. Perfusionszeit der A centralis retinae bei Normaldruckglaukom Erste Ergebnisse. Ophthalmologe 1994;91:595-601.

43 Tuulonen A, Airaksinen PJ. Optic disc size in exfoliative, primary open angle, and low-tension glaucoma. Arch Ophthalmol 1992;110:211-3.

44 Burk ROW, Rohrschneider K, Noack H, Völcker HE. Are large optic nerve heads susceptible to glaucomatous damage at normal intraocular pressure? A threeArch Clin Exp Ophthalmol 1992:230:552-60.

45 Jonas JB, Stürmer J, Papastathopoulos KI, Meier-Gibbons F, Dichtl A. Optic disc size and optic nerve damage in normal pressure glaucoma. Br f Ophthalmol 1995;79:1102-5. 